\title{
Multi-criteria planning model of engines parts additive manufacturing
}

\author{
Victoria Kokareva ${ }^{1,}{ }^{*}$, Anton Agapovichev ${ }^{1}$, Anton Sotov ${ }^{1}$, Vitaliy Smelov ${ }^{1}$, and Vadim \\ Sufiiarov ${ }^{2}$ \\ ${ }^{1}$ Samara State Aerospace University (National Research University), 443086 Moskovskoe sh. 34, \\ Russia \\ ${ }^{2}$ National Technology Initiative Center of Excellence in Advanced Manufacturing Technologies at \\ Peter the Great St. Petersburg Polytechnic University, 195251, St.Petersburg, Polytechnicheskaya, 29
}

\begin{abstract}
This paper reviews the state-of-the-art of an additive process planning methods amd models. Paper deals with the literature review of the planning process of additive manufacturing. The study also demonstrate multi-criteria planning model of engines parts additive manufacturing using multi-attributes decision-making problems approach.
\end{abstract}

\section{Introduction}

The choice of optimal process parameters in AM generally needs a high level of expertise and experience from the machine operator. The choice of AM process parameters such as part build orientation, or a non optimal design of support structures, can have drastic consequences on the performance of AM production, and more generally on the quality and sustainability of the manufacturing process.

Additive process planning (APP) is determining the sequence of set manufacturing operations needed to produce a given part with a correspond machine. Additive manufacturing (AM) pre-production processes consists of a set of standard operations : 3D modelling, orientation, supports, slicing and toolpath generation [1]. The most important step is the part orientation, planning process strategy influence on building time and surface quality. Computer Aided Process Planning (CAPP) helps to find an optimal model orientation according to different criteria such as minimization of supporting structures, building time or quality, printing cost. The main goal of CAPP is obtain solutions to the orientation optimization of multi-part production, where a group of parts in the same build time and chamber should be orientated simultaneously, according with the ultimate goal of minimizing the total build time and cost.

In order to determine the optimal build-up direction of a part for additive machine we take into account a variety of factors, such as: model height, volume of the necessary support structures, contact area between the nominal shape and the supports, surface roughness, build time, part cost, labour cost rate, material cost, etc. In this way, we could use genetic algorithm to obtain the optimum solution.

\footnotetext{
*Corresponding author: victoriakokareva@gmail.com
} 
The support generation technique should reduce wasted time and material in 3D printing by generating space-efficient branching support structures.

A tool-path generation algorithm provide geometrical accuracy, an adaptive speed of the additive nozzle/print head to address the geometrical characteristics of each layer and to identify the best slope degree of the zigzag tool-paths towards achieving the minimum build time.

Most authors agree that planning problems cannot be treated separately - a better understanding of the mutual relations between the parameters that govern these steps would make $3 \mathrm{D}$ printing more predictable, less error prone and would ultimately produce higher quality parts.

Today AM machines could build multiple parts simultaneously by placing multiple part slices within each building layer. In this case, we have difficulties of decision making in multi-part production context [2]: different parts, printing time, qualities, materials, requirements, available machines, materials, workers, resources.

\subsection{Process Planning for Additive Manufacturing}

Additive manufacturing process planning chain consist of several steps: stl. file generation, orientation determination, nesting, support generation, slicing, scanning path generation, layer building, post processing. Engines parts production is multiple parts manufacturing that why the task of process planning is to reduce the complexity, the printing time and post processing based on process planning strategy. Different engines parts are featured different production requirements, e.g. lead time, cost, and quality. Therefore, production manager should solve two conflicting decision optimizations tasks: first is the selection of the required AM process and equipment with evaluation of build time, cost and quality, the second is orientation parts optimization, work space planning, support generation, slicing, tool path planning. AM shops have different AM machines and software tools (SLM, DMD, etc.) which characterized unique algorithms for orientation optimization and workspace planning in the multiple parts production context. In the multiple parts production context the total build time, cost and quality depend on the combination of part's build orientations and space planning. Hence, the general planning tasks in additive multiple parts manufacturing is developing the method of AM build platform generation based on multi-criteria planning model.

The chain of AM build platform generation is shown in Figure 1.
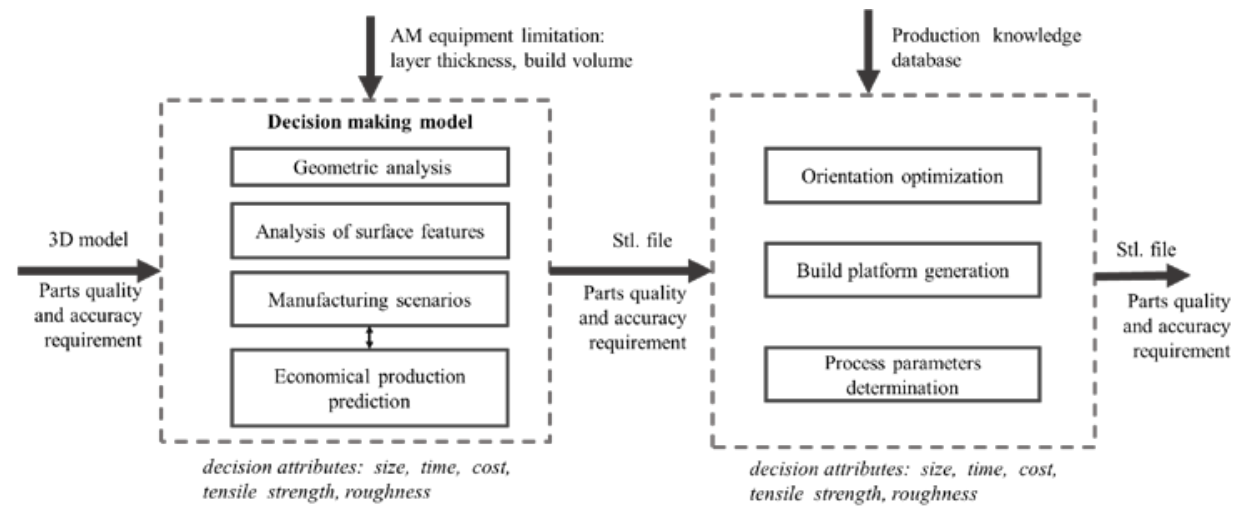

Fig. 1. The chain of AM build platform generation. 
Geometric analysis refers to analyze the size and shape, complexity of a part and key geometric features of a part. Parts which size are exceed the build volume cannot be included in build process on this AM machine.

The limited layer thickness of AM machine lead difficulty to print some shape features with very small sizes. Unfortunately, the AM processes cannot guarantee the shape accuracy of some features like long tiny holes or cylinders or large planes, shells, etc.

If a part can be printed by chosen AM machine with correspond AM process there are next steps of planning concern to manufacturing scenarios. The output of this task is the rank of alternative AM process - manufacturing scenarios which are obtained by a decision making model.

The economical production prediction is concern of build time and cost of printing.

A key problematic feature of AM process planning is to identify the possible AM machine (technology) using algorithm, which eliminates and generate potential alternatives according to the checking of decision attributes, e.g. size, time and cost, tensile strength, roughness. Moreover, the determination of the build time and cost for individual parts that are building simultaneously is a big challenge. Using mathematical/numerical methods to compute production quality, time and cost estimation is relatively low due to the large computation. Another method is experimental results evaluation, but it need the production knowledge base on large quantity of experimental results.

The interconnection between Manufacturing scenarios and Economical production prediction means that we have different AM manufacturing scenarios and processing specifications are stored in production database, and build time and cost prediction methods, which based on production knowledge.

Orientation optimization is one of the key planning tasks to guarantee the parts quality. We should combine alternative orientation of parts in one build to reduce support volume, total cross section area. It is a very complex combinatorial task - multi-objective NPcomplete problem.

The aim of build platform workspace planning is maximizing parts nesting in machine build volume with production quality guarantee. The output of orientation optimization is the input of the workspace planning. It is also NP-compete task. The output of this task is a group of positioned parts that can be sent to machine planning tasks, support generation, slicing and scanning-path planning. Nesting decision can based on application of a genetic algorithm for searching an optimal nesting solution with minimum total overlap area.

To simplify multiple parts production we can use clustering algorithm: changing the part sequence according to their "similarity" based on production knowledge and measuring model.

The main process factors, such as laser power, scanning speed, layered thick and scan spacing affect on AM parts quality [9], [10].

\section{Multi-criteria planning model}

The problem of AM planning is consists of a set of AM machines, where each AM machine has different specifications, including operation cost, production efficiency and maximum supported area and height. There exists a set of parts with different volumes, heights and production areas as determined by the requirements. The parts will be allocated to AM machines and then grouped as different sets of build platforms by considering the production time and cost. The jobs then will be performed in the AM machines according to the production schedule of each AM machine [3]. 


\subsection{Multi-criteria planning model of engines parts additive manufacturing}

AM engines parts quality is characterized by the strength, precision and deformation. Figure 2 shows the influence of AM process parameters on the parts behavior [4].

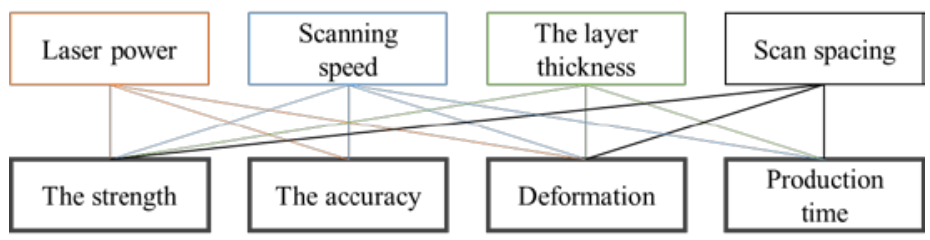

Fig. 2. The influence of a reasonable process parameters.

According to effect of AM process parameters on the forming quality, strength, accuracy, deformation and the production time, we obtain the matrix $\mathrm{G}$ of AM process parameters and the matrix $C$ for various reasonable parameters: $C_{1}-$ the strength, $C_{2}-$ the accuracy, $\mathrm{C}_{3}-$ deformation, $\mathrm{C}_{4}$ - production time (Fig. 3 ).

$$
\begin{gathered}
G=\left(\begin{array}{cccc}
1 & 2 & 2 & 3 \\
1 / 2 & 1 & 1 & 2 \\
1 / 2 & 1 & 1 & 2 \\
1 / 3 & 1 / 2 & 1 / 2 & 1
\end{array}\right) C_{1}=\left(\begin{array}{cccc}
1 & 1 & 1 / 2 & 1 / 2 \\
1 & 1 & 1 / 2 & 1 / 2 \\
2 & 2 & 1 & 1 \\
2 & 2 & 1 & 1
\end{array}\right) C_{2}=\left(\begin{array}{ll}
1 & 1 \\
1 & 1
\end{array}\right) \\
C_{3}=\left(\begin{array}{cccc}
1 & 1 & 1 / 3 & 1 / 2 \\
1 & 1 & 1 / 3 & 1 / 2 \\
3 & 3 & 1 & 2 \\
2 & 2 & 1 / 2 & 1
\end{array}\right) C_{4}=\left(\begin{array}{ccc}
1 & 1 / 3 & 1 / 2 \\
3 & 1 & 2 \\
2 & 1 / 2 & 1
\end{array}\right)
\end{gathered}
$$

Fig. 3. Additive manufacturing matrix.

The target goal of multi-criteria planning model of engines parts additive manufacturing is minimization of build time.

The algorithm (1) allows calculating the build time according with the proportion part cross-section or part height and the amount of slices layers. The algorithm suggests optimizing the use of building space by simultaneously building up as much geometries with similar part height as possible [5].

$$
\sum_{i} T_{\text {build }}=a_{0}+a_{1} \cdot N_{L}+a_{2} \cdot V_{\text {tot }}+a_{3} \cdot S_{\text {SUPPORT }}+a_{4} \cdot \sum_{i} N_{i}+a_{5} \cdot S_{\text {tot }}
$$

where $T_{\text {build }}$ - building time of part with $i_{\text {th }}$ geometry; $\mathrm{a}_{0} \ldots \mathrm{a}_{5}$ - regression coefficients; $N_{l}$ - number of layers; $V_{\text {tot }}$ - total volume of the build job, $\mathrm{mm}^{3} ; \mathrm{S}_{S U P P P O R T}$ - total surface area of the support structures, $\mathrm{mm}^{2} ; N_{i}$ - quantity of parts with $\mathrm{i}_{\text {th }}$ geometry; $S_{\text {tot }}$ - total surface area of the build job, $\mathrm{mm}^{2}$.

During laser melting the amount of processed parts varies from layer to layer, since the parts heights are different. Therefore, time fraction resulting from the total amount of layers has to be allocated to each part on a layer-wise basis. Otherwise, smaller parts will be wrongly cost estimated because of the simultaneous build up. To avoid this, regression coefficients were developed.

The algorithm suggests follow steps: 
1. Generation of parts ordering by increasing height.

2. Calculation of the melting time resulting from the amount of layers up to the smallest part height and divide it equally on all parts.

3. Choose of the next taller part.

4. Calculation of the time fraction from the previous to the height of the actual part.

5. Division of the calculated time equally on all parts with a part height equal or greater than actual part's height.

6. Repetition of steps 3 to 5 until all parts are processed.

Therefore, the build time of one part will be calculated:

$$
T_{\text {build }}\left(P_{i}\right)=\frac{a_{0}}{\sum_{i} N_{i}}+P_{i} \cdot T_{L}+a_{2} \cdot V\left(P_{i}\right)+a_{3} \cdot S_{\text {SUPPORT }}\left(P_{i}\right)+a_{4}+a_{5} \cdot S\left(P_{i}\right)
$$

where $P_{i}$ - part with $i_{\text {th }}$ geometry; $T_{L}$ - layer-dependent melting; $V$ - volume of the part; $S$ - surface area of the part.

Different combinations of parts in a build platform will lead to different times, as the total time is characterized by the total volume and maximum height of parts assigned to the build platform.

\subsection{Implementation of multi-criteria planning model}

AM process planning is based on knowledge expert system of multi-attributes decisionmaking problems, such as the selection of AM machines or processes, selection of AM materials and determination of a set of AM parameters and parts [6]. When we use AM techniques for engines parts manufacturing we should know how to select the best combination of parts and materials, machines according to end-use requirements taking into account the built orientation. In this way the planning model eliminate and select the alternatives combination of AM technical parameters, which satisfy parts quality and minimization build time, 2.1, Fig.2. The alternatives combination of AM technical parameters and target goal are presented as knowledge vector with their attributes [7].

Vector $V_{A M}=(A, R, S, C, T)$. For each $A M$ machines vector $V_{A M}$ will consist of original attributes: $\mathrm{A}$ - accuracy $/ \mu \mathrm{m}, \mathrm{R}$ - surface roughness $/ \mu \mathrm{m}, \mathrm{S}-$ tensile strength $/ \mathrm{MPa}$, $\mathrm{C}-$ cost of material/ Rub, $\mathrm{T}-$ build time/hour. $\mathrm{V}_{\text {goal }}=\min _{\text {build }}[8]$. The model investigate the relation between alternatives and target goal. This means the alternative is closer to the target goal and their relation is tighter. The task of model calculation is determination the disparity between alternatives and the target goal, selecting the alternative which has the least disparity. Hence, if we have two different AM machines, e,g, SLM and DMD, there are two vectors :

$$
\mathrm{V}_{1}=\left(\varepsilon_{\mathrm{i}}(1), \varepsilon_{\mathrm{i}}(2), \ldots \varepsilon_{\mathrm{i}}(\mathrm{n})\right), \mathrm{V}_{2}=\left(\varepsilon_{\mathrm{j}}(1), \varepsilon_{\mathrm{j}}(2), \ldots \varepsilon_{\mathrm{j}}(\mathrm{n})\right) \text {, where } \varepsilon_{\mathrm{i}}(\mathrm{n}) \text { and } \varepsilon_{\mathrm{j}}(\mathrm{n}) \text { are }
$$
indexes of atrributes disparity. For example, $V_{A}=\left(A_{1}, A_{2}\right), V_{R}=\left(R_{1}, R_{2}\right)$, where $A_{1}, A_{2}-$ the value of accuracy/ $\mu \mathrm{m}$ for corresponding machines, $R_{1}, R_{2}$ - the value of surface roughness $/ \mu \mathrm{m}$. By applying the mean operator attribute vectors can be obtained: $\mathrm{DV}_{\mathrm{A}}=\left(\mathrm{dA}_{1}, \mathrm{dA}_{2}\right), \mathrm{DV}_{\mathrm{R}}=\left(\mathrm{dR}_{1}, \mathrm{dR}_{2}\right)$, where $\mathrm{D}$ is a mean operator, and for attribute $\mathrm{A}$, the process is like that:

$$
d A_{i}=\frac{A_{i}}{A(e)}=\frac{A_{i}}{1 / 2 \sum_{i}^{2} A_{i}}, A(e) \text { is the mean value of vector } \mathrm{V}_{\mathrm{A}} \text { 's elements. Then the }
$$

alternative vectors of SLM $-\mathrm{V}_{\mathrm{AM} 1}=\left(\mathrm{dA}_{1}, \mathrm{dR}_{1}\right)$, and DMD $-\mathrm{V}_{\mathrm{AM} 2}=\left(\mathrm{dA}_{2}, \mathrm{dR}_{2}\right)$, while $V_{\text {goal }}=\left(\mathrm{dA}_{\mathrm{opt}}, \mathrm{dR}_{\mathrm{opt}}\right)$. We should assigne the weight or rank of attributes vector: 
$\omega \mathrm{V}_{\mathrm{AM} 1}=\left(\omega_{1} \mathrm{dA}_{1}, \omega_{2} \mathrm{dR}_{1}\right)$, and DMD $-\omega \mathrm{V}_{\mathrm{AM} 2}=\left(\omega_{1} \mathrm{dA}_{2}, \omega_{2} \mathrm{dR}_{2}\right), \omega_{1}$ and $\omega_{2}-$ is the assigned set of weights for the related attributes. Likewise, the target goal vector can be interpreted by all of the best values of each attribute from the reconstructed alternatives, as $\mathrm{V}_{\text {goal }}=\left(\omega_{i}{ }^{j} \mathrm{dA}_{\mathrm{opt}}, \omega_{i}{ }^{j} \mathrm{dR}_{\mathrm{opt}}\right), i-$ number of attributes, $j-1,2$. Than we should evaluate the each atrributes disparity and compare with the target goal vector by similarity model. The ranking of the AM process is based on the general index values, which means that the one who has the largest index value would be the most recommended alternative among the finite choices.

\section{Summary and conclusions}

This paper presents a study on AM process planning model multiple engines parts production. This is just the first result and further research will be carried out and concern with developing making decision model for SLM production planning. In order to decrease the AM time and cost there are necessary to improve optimization algorithm, develop prediction model base.

\section{References}

1. M. Livesu, M. Attene, M. Spagnuolo, B. Falcidieno, A study of the state of the art of process planning for additive manufacturing, , Istituto di Matematica Applicata e Tecnologie Informatiche, May 2016.

2. Y. Zhang, A. Bernard, AM feature and knowledge based process planning for additive manufacturing in multiple parts production context, 2014.

3. Q.Li et al., Production planning in additive manufacturing and $3 D$ printing, Computers and Operations Research 83, 157-172 (2017)

4. Yang Laixia, Deng Jun, Li Dichen, Bai Yang, The multi-objective optimization for the SLS process parameters based on analytic Hierarchy Process, International science index, mechanical and mechatronics engineering Vol:6, No:7 (2012)

5. L. Rickenbacher, Adriaan B. Spierings, Konrad Wegener, An integrated cost-model for selective laser melting (SLM), Rapid Prototyping Journal 19(3), April 2013.

6. Smelov, V.G., Kokareva, V.V., Malykhin, A.N., Lean organization of additive manufacturing of aircraft purpose products, International Journal of Engineering and Technology, Volume 6, Issue 5 (2014).

7. Yicha Zhang, Alain Bernard, An integrated decision-making model for multi-attributes decision-making (MADM) problems in additive manufacturing process planning, Rapid Prototyping Journal, Volume 20, Number 5 (2014).

8. Agapovichev, A.V., Sotov, A.V., Kokareva, V.V., Smelov, V.G., Kyarimov, R.R., Study of the structure and mechanical characteristics of samples obtained by selective laser melting technology from VT6 alloy metal powder, International Journal of Nanomechanics Science and Technology 8(4), p. 323-330 (2017).

9. Sufiiarov V.Sh., Popovich A.A., Borisov E.V., Polozov I.A, Masaylo D.V., Orlov A.V., The Effect of Layer Thickness at Selective Laser Melting, Procedia Engineering 174, pp. 126-134 (2017).

10. Popovich A.A., Sufiiarov V.Sh., Borisov E.V., Polozov I.A, Masaylo D.V., Design and manufacturing of tailored microstructure with selective laser melting, Materials Physics and Mechanics 38, pp. 1-10 (2018). 